http://jmscr.igmpublication.org/home/

ISSN (e)-2347-176x ISSN (p) 2455-0450

crossref DOI: https://dx.doi.org/10.18535/jmscr/v7i11.154

Journal Of Medical Science And Clinical Research

IGM Publication

An Official Publication of IGM Publication

\title{
Serum Magnesium Level in Acute Exacerbation of Chronic Obstructive Pulmonary Disease
}

\author{
Authors \\ Dr Parimala Sundari Seetharaman ${ }^{1}$, Dr Arun Gnanasekaran ${ }^{2}$ \\ ${ }^{1}$ Associate Professor, Department of Medicine, Kilpauk Medical College, Chennai-10 \\ *Corresponding Author
}

Dr Arun Gnanasekaran

\begin{abstract}
Aim: To establish association of COPD and its exacerbations with S. Magnesium level.

Introduction: COPD is a long disease characterized by chronic obstruction of airflow that interferes with normal breathing and is not fully reversible. This study is to establish association of COPD with S. Magnesium level.

Materials \& Methods: It is a case control study, data collected from April 2018 to September 2018, for 6 months. Study population were patients $>18$ years of age.

Results: The incidence of hypo magnetic present on $81.1 \%$ of patients with Acute exacerbation (case), as compared to $0.01 \%$ of patients with stable COPD (control) with main Serum Magnesium of $1.57 \mathrm{mg} / \mathrm{dl}$ in Acute Exacerbation of COPD (case) as compared to mean Serum Magnesium of $2.10 \mathrm{mg} / \mathrm{dl}$ in stable COPD (control). This difference of mean values of S. Magnetism in COPD exacerbation and stable COPD patients was significant with $P<0.0005$.

Conclusion: In our study, hypo magnetic is also exacerbations episode in COPD patients.

Keywords: Bronchospasm, Exacerbation, Hypomagnesenic, S. Magnesium.
\end{abstract}

\section{Introduction}

Chronic obstructive pulmonary disease (COPD) is a lung disease characterized by chronic obstruction of lung airflow that interferes with normal breathing and is not fully reversible.

COPD is not simply a "smoker's cough" but an under-diagnosed, life-threatening lung disease. Chronic obstructive pulmonary disease (COPD) is leading cause of morbidity and mortality worldwide. Currently ranked as $5^{\text {th }}$ leading cause of death worldwide and predicted $3^{\text {rd }}$ upto 2020, it represents an important public health challenge that is both preventable and treatable.

As a result, there is a mounting interest not only in designing optimal COPD treatment approaches but also in preventing its exacerbations. Magnesium $\left(\mathrm{MG}^{+2}\right)$ is an intracellular cation which is involved in the regulation of bronchial tone, mast cell secretion, neuromuscular activity and respiratory muscle function.

Since magnetism is involved in muscle tone, therefore a decrease in magnesium in level in COPD patients causes bronchial muscle contraction.

A growing body of evidence suggests that $\mathrm{Mg}^{+2}$ deficiency contributes to exacerbations of asthma and, as a corollary, that $\mathrm{Mg}^{+2}$ is useful in alleviating bronchospasm in these patients. Although the precise mechanism of this action is unknown, it has been suggested that $\mathrm{Mg}^{+2}$ plays a role in the 
maintenance of airway patency via relaxation of bronchial smooth muscle.

Bronchospasm is a contributing factor in their inability to clear secretions. This may result in reduced pulmonary gas exchange with consequences such as decreased quality of life and repeated hospitalization.

Thus, $\mathrm{Mg}^{+2}$ may have a role in maintaining disease stability in COPD patients. This study is to establish association with serum magnesium level and exacerbations.

\section{Materials and Methods}

Study Design: Case control study (Descriptive)

Study Period: Data collection done period of 6 months from April 2018 to September 2018.

Place of Study: Government Kilpauk Medical College and Hospital, Chennai-10.

Study Population: Patients $>18$ years of age.

Case Group: The case group included subjects who presented with an exacerbation of COPD requiring hospitalization in the department of internal medicine.

Control Group: The patients attending outpatient department with stable COPD for routine check-up are included in control group.

Sample Size: Case-58, Control-58, Total-116

\section{Inclusion Criteria}

\section{Case and Control Groups}

Patients in case and control groups were diagnosed as having COPD based on dynamic pulmonary function test results (ratio of 1-sec forced expiratory volume, FEV1/ forced vital capacity, FVC $<70$ ), according to the European Respiratory Society Task Force recommendations.

\section{Case Group}

Patients in case group were diagnosed as having acute exacerbation based on the criteria of Anthonisen et al, i.e. either presence of shortness of breath, or severe cough with or without increased sputum volume.

\section{Exclusion Criteria}

A. Patients with following associated conditions which can be a separate risk factor for electrolyte imbalance were excluded.

1. Gastrointestinal disease: Malabsorption syndrome, ulcer disease, pancreatitis and severe diarrhea.

2. Pregnancy and lactation.

3. Hormonal disease: diabetes mellitus, hypothyroidism, hyperthyroidism.

4. Renal failure.

5. Drugs: thiazide diuretics, loop diuretics.

6. Malignancy.

7. Alcoholism.

B. Patients with pulmonary embolism and pneumothorax.

C. Patients with acute exacerbation of bronchiectasis.

\section{Methodology}

Collection of Data: The patients' blood sample was drawn and sent to analysis for serum magnesium level patients were analysed for pulmonary function test and was staged are to GOLD Criteria.

\section{Statistical Analysis}

\section{Statistical Method}

1) Percentage analysis were used for categorical variables.

2) Mean and S.D were used for continuous variables.

3) To find the significant difference between the bivariate samples in Independent groups the unpaired sample t-test was used.

\section{Results}

Fig.1 : Overall age distribution \%

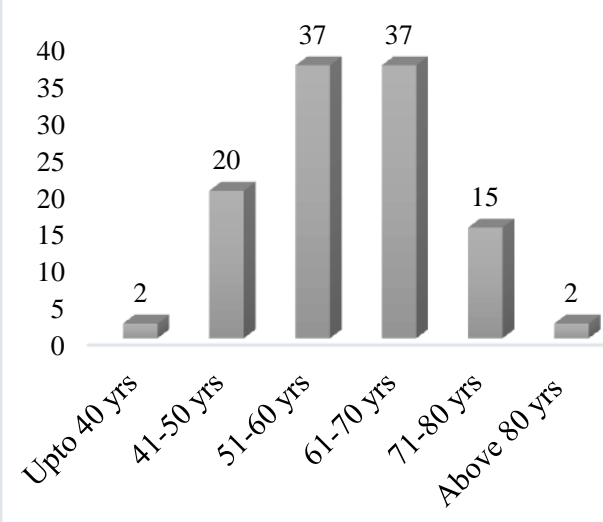


The overall age distribution of the study population as follows:

Upto 40 years of age 2 people were included in the study, 41 to 50 years of age 20 were included, 51 to 60 years of age 37 were included, 61 to 70 years of age 37 were included, 71 to 80 years of age 15 were included and more than 80 years of age 2 were included. Majority of the study group belongs to the age group of 51 to 70 years which indicate the most common aetiology is of chronic exposure to irritants such as tar or indoor pollutants or particulate matter in the inhaled air.

Fig.2: Gender distribution

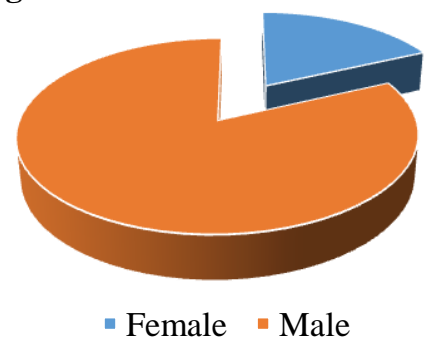

In this study, out of 116 patients enrolled 95 patients were males and 21 patients were females, with male - female ratio $81.9 \%$ - $18.1 \%$ (i.e., around $4: 1$ ).

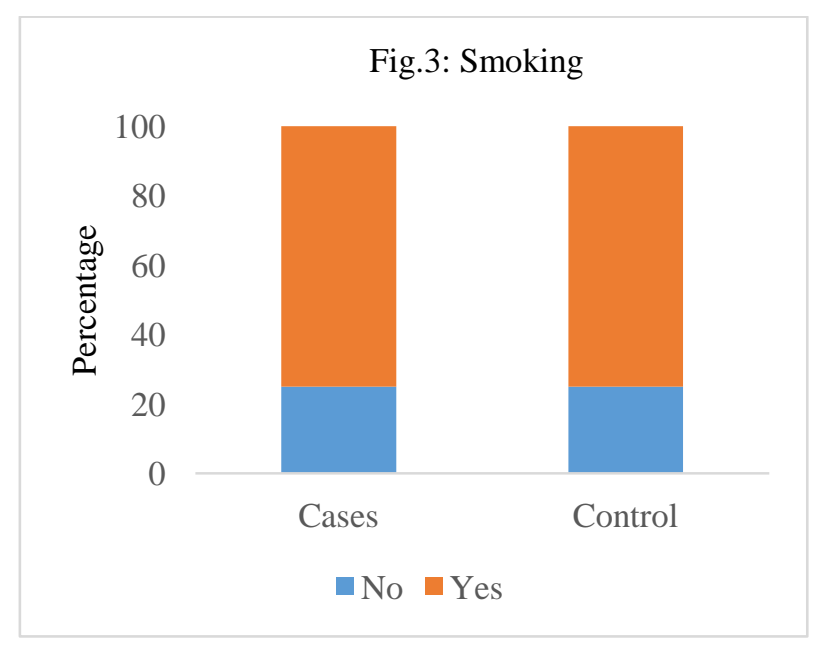

In this study, out of 116 patients enrolled 95 patients were males and 21 patients were females, among them $75 \%$ of COPD patients were chronic smokers, $25 \%$ patients were non-smokers among the patients studied.
Table 1: Comparison of stages of COPD and Sr. $\mathrm{Mg}^{2+}$ levels

\begin{tabular}{|c|c|c|c|c|}
\hline \multicolumn{2}{|c|}{} & \multicolumn{2}{c|}{ Groups } & \multirow{2}{*}{ Total } \\
\cline { 3 - 4 } \multicolumn{2}{|c|}{} & Cases & Control & \\
\hline STAGING 1 & Count & 2 & 2 & 4 \\
\hline & $\begin{array}{c}\text { \% within } \\
\text { Groups }\end{array}$ & $3.4 \%$ & $3.4 \%$ & $3.4 \%$ \\
\hline 2 & Count & 29 & 30 & 59 \\
\hline 3 & $\begin{array}{c}\% \text { within } \\
\text { Groups }\end{array}$ & $50.0 \%$ & $51.7 \%$ & $50.9 \%$ \\
\hline & Count & 23 & 21 & 44 \\
\hline & $\begin{array}{c}\text { \% within } \\
\text { Groups }\end{array}$ & $39.7 \%$ & $36.2 \%$ & $37.9 \%$ \\
\hline Total & $\begin{array}{c}\text { Count } \\
\text { Groups }\end{array}$ & 4 & 5 & 9 \\
\hline & Count & 58 & 58 & 116 \\
\hline & $\begin{array}{c}\% \text { within } \\
\text { Groups }\end{array}$ & $100.0 \%$ & $100.0 \%$ & $100.0 \%$ \\
\hline
\end{tabular}

Fig.4: Staging

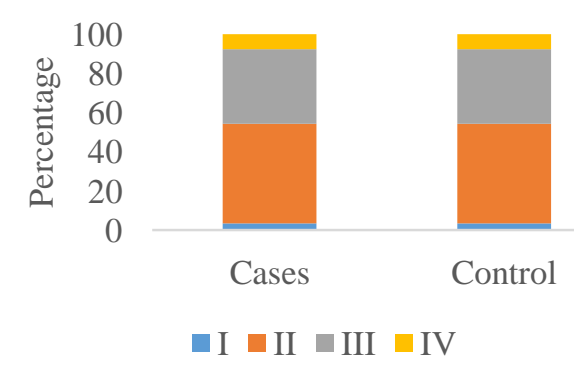

In this study,

$3.4 \%$ patients were in stage I,

$50.9 \%$ patients were in stage II,

$37.9 \%$ patients were in stage III and

$7.8 \%$ patients were in stage IV.

Table 2

\begin{tabular}{|l|c|c|c|c|c|}
\hline Groups & $\mathrm{N}$ & Mean & $\begin{array}{c}\text { Std. } \\
\text { Devia- } \\
\text { tion }\end{array}$ & $\begin{array}{c}\text { Std. } \\
\text { Error } \\
\text { Mean }\end{array}$ & $\begin{array}{c}\text { P } \\
\text { VALUE }\end{array}$ \\
\hline $\begin{array}{l}\text { S. Mg } \\
\text { Cases } \\
\text { LEVEL }\end{array}$ & 58 & 1.5731 & .17987 & .02362 & $<0.0005$ \\
\hline Control & 58 & 2.1022 & .21958 & .02883 & \\
\hline
\end{tabular}

Fig.5: $\mathrm{Mg}$ Level

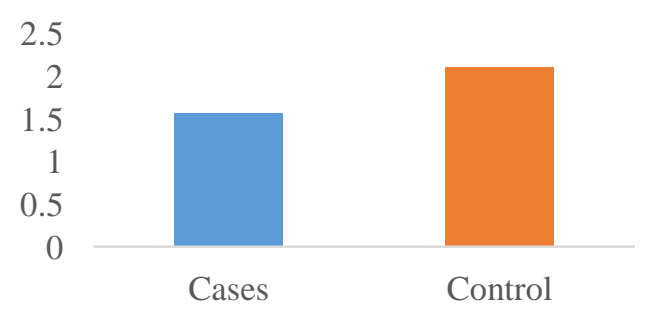


The incidence of hypomagnesaemia present in $81.1 \%$ of patients with acute exacerbation of COPD (case) as compared to $0.01 \%$ patients with Stable COPD (control), with the mean serum magnesium of $1.57 \mathrm{mg} / \mathrm{dl}$ in acute exacerbation of COPD (case) as compared to mean serum magnesium of 2.10 $\mathrm{mg} / \mathrm{dl}$ in Stable COPD (control). This difference of mean values of serum magnesium in COPD exacerbation and stable COPD patients was significant with $\mathrm{p}$ value of $<0.0005$.

\section{Discussion}

There is growing awareness of serum magnesium level in pulmonary diseases. Much of the impetus for recognition of magnesium as both risk factor and potential therapeutic agent in patients with COPD comes from relatively well established role of magnesium in the treatment of acute asthma Magnesium disturbance is a well-known abnormality seen in patients with pulmonary disease.

In our study the maximum numbers of patients with exacerbation were having predominant stage II and stage III according to GOLD staging criteria for COPD3.

About $80.8 \%$ of the patients with hypomagnesaemia were having stage II and stage III disease and 7.8\% were in stage IV. In our study, there was significant correlation was present between hypomagnesaemia and GOLD staging II and stage III ( $\mathrm{p}=0.001$ and $\mathrm{p}=0.0006$ respectively), with nonsignificant correlation between hypomagnesaemia and stage I.

Our patient did not receive any replacement therapy for reduced serum magnesium level. This can be explained by either correction of hypoxia, treatment of infection or avoidance of drugs precipitating hypomagnesaemia would correct hypomagnesaemia.

The frequent use of methylxanthines, $\beta 2$ - agonist inhalers and $\beta 2$ - agonist oral preparation has been described as a cause of Magnesium depletion and hypomagnesaemia. Hypomagnesaemia in patients with acute pulmonary disease has also been related to a result of severe infections, inappropriate secretion of anti-diuretic hormone (ADH), antibiotic administration (aminoglycosides) etc.

The potential mechanism for the direct relaxing effects of magnesium on bronchial smooth muscles include calcium channel blocking properties, inhibition of cholinergic Neuro-Muscular Junction transmission with decreased sensibility to the depolarizing action of acetylcholine, stabilization of mast cells and $\mathrm{T}$ lymphocytes and stimulation of nitric oxide and prostacycline.

\section{Conclusion}

In our study, Hypomagnesaemia is associated with exacerbation episodes in COPD patients.

Further study on correction of hypomagnesaemia in stable and exacerbations of COPD patients in preventing and treating exacerbations and studies about exacerbation frequencies in patients with normomagnesaemia and hypomagnesaemia in large population, will be informative and useful. 